\title{
Möglichkeiten und Grenzen der Messung interkultureller Kompetenz
}

ABSTRACT. There is no doubt that a cultural dimension is a part of the foreign language teaching. Still, the necessity of assessing learners' intercultural competence seems to be overlooked. The following article examines possibilities of assessing intercultural competence implying not only quantification and judgment but also reflecting the effectiveness of intercultural foreign language teaching. The paper gives also an insight into the limits of such kind of assessment.

\section{EINFÜHRUNG}

Die Bedeutung interkultureller Kompetenz und interkulturellen Lernens und Lehrens ist in der Fremdsprachendidaktik weitgehend anerkannt und unter dem Begriff interkulturelle Fremdsprachendidaktik subsumiert worden. Die Auseinandersetzung mit den Bedeutungsfacetten der vorstehenden Begriffe und ihrer Ausprägungen ist bereits seit langem Teil des glottodidaktischen Diskurses. Das Thema Messung interkultureller Kompetenz ist hingegen bis dato nur selten Inhalt wissenschaftlicher Veröffentlichungen. Dies ist insofern überraschend, als die interkulturelle Fremdsprachendidaktik den systematischen Einbezug von Fremdperspektiven in die Vermittlung der fremden Sprache und Kultur auf folgenden Ebenen fordert (vgl. Müller 1994: 158, Hervorhebung: A.B.):

- in der Bestimmung der Lernziele,

- in der Auswahl der Lerninhalte,

- in den präsentierten Lernverfahren,

- in der Art der Lernkontrolle.

Den Anforderungen der drei ersten Aspekte widmen sich mehrere Veröffentlichungen. Der letzte Bereich kann zur Zeit nur als Problem dargestellt 
werden. Die umfassende Neuorientierung des Denkens, die interkulturelles Lehren und Lernen mit sich bringt, wäre - wie es scheinen mag - kaum mit den gängigen Testverfahren zu überprüfen. Es gilt allerdings dieser Anforderung der interkulturellen Fremdsprachendidaktik gerecht zu werden, da Lernende zur Zeit nur in den vier klassischen Fertigkeitsbereichen ihr Sprachwissen überprüfen können. Deshalb muss nach Strewe (2005) die Frage gestellt werden: Gibt es Methoden zur Erfassung oder Messung der interkulturellen Kompetenz? Welche von den gängigen Instrumenten oder Bewertungsskalen wären hierzu geeignet? Können Einstellungen bzw. Akzeptanz/Respekt gegenüber anderen Kulturen überhaupt beurteilt und bewertet werden? Gibt es u.a. im universitären Lehrbetrieb neutrale und objektive Maßstäbe zur Bewertung interkultureller Kompetenz?

In dem vorliegenden Beitrag sollen die Fragen näher erörtert werden, dabei wird davon ausgegangen, wie interkulturelle Kompetenz in ihrer Komplexität hinsichtlich der Messung aufzufassen ist und inwiefern das bestehende Instrumentarium der Leistungsmessung in der Fremdsprachendidaktik der Messung interkultureller Kompetenz gerecht bzw. nicht gerecht werden kann. Darüber hinaus soll auf die Besonderheiten und Schwierigkeiten der Messung interkultureller Kompetenz näher eingegangen werden.

\section{ZUR KONSTRUKTION DER INTERKULTURELLEN KOMPETENZ IM HINBLICK AUF IHRE MESSUNG}

Eine valide Leistungsmessung bedarf zunächst der Aufstellung von Kriterien, nach denen ein Phänomen evaluativ erfasst werden kann. Den Ausgangspunkt in dem vorliegenden Fall bildet ein entsprechendes Modell der interkulturellen Kompetenz, von welcher Byram (1997: 73) ein sehr detailliertes Bild liefert. Das ist an sich zu begrüßen, es erschwert allerdings unter Umständen die Messung der interkulturellen Kompetenz. Der entscheidende Vorteil dieses Modells liegt jedoch darin, dass es die für die Fremdsprachendidaktik relevanten Komponenten viel stärker berücksichtigt als die anderen bekannten Modelle (vgl. u.a. Bolten 2001: 214; Thomas 2003: 145). Während sich z.B. das Modell von Bolten stark an der Effektivität von Wirtschaftskontakten orientiert und das Modell von Thomas an der allgemeinen Persönlichkeitsentwicklung, definiert Byram (vgl. auch Sercu 2004: 74) konkrete Anforderungen für die Fremdsprachendidaktik und misst der Sprache die ihr zukommende Bedeutung bei. Dabei wird ein Aspekt betont, der bei den anderen Modellen nach unserer Ansicht zu kurz kommt: Obwohl es sich um die Messung interkultureller Kompetenz im engeren Sinne handelt, darf der holistische Blick auf die interkulturelle kommunikative Kompetenz nicht verloren gehen. Dies ist nach Ansicht der Autorin nur bei dem Modell von Byram gegeben. 
Das Modell der interkulturellen Kompetenz nach Byram (1997: 70-73) ist also in der interkulturellen kommunikativen Kompetenz verankert, die hier als ein Zusammenspiel folgender charakteristischer Komponenten ausgewiesen wird:

- linguistische Kompetenz,

- soziolinguistische Kompetenz,

- Diskurskompetenz,

- interkulturelle Kompetenz.

Die von uns fokussierte interkulturelle Kompetenz sensu stricto erfordert die Darstellung von fünf, mit kommunikativer Kompetenz verflochtenen Dimensionen, die Byram (1997: 34) 5 savoirs genannt hat. Diese 5 savoirs sind als integrierte, mit kommunikativer Kompetenz verflochtene Dimensionen interkultureller Fähigkeit zu betrachten und können wie folgt umschrieben werden: savoir être als Einstellungen, savoir comprendre als Verstehen unter Nutzung kulturellen Wissens, savoir apprendre/faire als Fähigkeiten zur Organisation von Erkenntnisgewinn und Interaktion in einer zunächst fremden Umgebung und savoir s'engager als die kritische Beurteilung der Eigen- und Fremdkultur (vgl. Boeckmann 2006).

Sercu (2004: 74) hat dieses Modell weitgehend beibehalten, jedoch zur besseren Übersichtlichkeit in einem Punkt grundsätzlich modifiziert. Sie hat das Modell von Byram (1997) eindeutig in einen Rahmen der "Ebenen der interkulturellen Kompetenz" (Tabelle Nr. 1) eingebettet, was tabellarisch wie folgt dargestellt werden kann:

Tabelle Nr. 1: Ebenen interkultureller Kompetenz nach Sercu (2004: 74)

\begin{tabular}{|c|c|}
\hline Ebenen der IK & Allgemeine Qualifikationen \\
\hline \multirow{4}{*}{ Wissen } & allgemeine Kulturkenntnisse \\
\hline & Wissen über die eigene und fremde Kultur \\
\hline & Kenntnisse der individuellen und sozialen Interaktion \\
\hline & $\begin{array}{l}\text { Einsicht in die Art und Weise, auf die Sprache auf ihrer verbalen } \\
\text { und paraverbalen Ebene von Kultur beeinflusst wird }\end{array}$ \\
\hline Einstellungen & $\begin{array}{l}\text { Offenheit und Bereitschaft, die eigene Perspektive zu relativieren } \\
\text { Neugierde }\end{array}$ \\
\hline \multirow{5}{*}{ Handlungen } & $\begin{array}{l}\text { Interpretationsvermögen im Hinblick auf die fremde Kultur und } \\
\text { ihren Bezug auf die eigene Kultur }\end{array}$ \\
\hline & Fähigkeit, die Realität und Interaktion zu beobachten \\
\hline & $\begin{array}{l}\text { Fähigkeit, Kenntnisse über eine fremde Kultur zu erwerben und } \\
\text { diese in der Kommunikation und Interaktion angemessen an- } \\
\text { zuwenden }\end{array}$ \\
\hline & $\begin{array}{l}\text { Fähigkeit, metakognitive Strategien einzusetzen: über eigenes } \\
\text { Lernen zu reflektieren }\end{array}$ \\
\hline & $\begin{array}{l}\text { kritisches Urteilsvermögen im Hinblick auf die eigene und frem- } \\
\text { de Kultur }\end{array}$ \\
\hline
\end{tabular}


Zu den Einstellungen zählt Sercu (ebd. auch Byram 1997: 34-38): Wissensbegierde und Offenheit sowie die Bereitschaft, Misstrauen gegenüber anderen Kulturen abzubauen und auf das egozentrische Bild der eigenen Kultur zu verzichten. Wissen bezieht sich auf die Kenntnisse bzw. auf das Bewusstsein über die Differenzen zwischen Kulturen bzw. sozialen Gruppen sowie über allgemeine Prozesse, denen unsere sozialen Interaktionen unterliegen (Wahrnehmung der Geschichte, Institutionen, Nationalgedächtnis etc.). Diese Komponente umfasst auch das Wissen über Kulturausdrücke und -verhalten im eigenen Kontext sowie im Kontext des Gesprächspartners. Allgemein kann gesagt werden, dass diese Komponente mit der Fähigkeit verbunden ist, Kulturen im geschichtlichen, geographischen und sozialen Kontext zu vergleichen. Dies impliziert theoretisches Wissen.

Handlungen umfassen das Interpretationsvermögen und das Vermögen zur Aufstellung von gegenseitigen Bezügen (eng. skills of interpreting and relating) z.B. hinsichtlich von Dokumenten und Ereignissen aus der eigenen und der fremden Kultur. Damit ist die Fähigkeit verbunden, Konflikte zu identifizieren und zu interpretieren und zwar insbesondere solche, die auf Ethnozentrismus zurückzuführen sind. Hierzu gehört auch die Fähigkeit, die Realität und Interaktion zu beobachten (eng. skills of discovery and interaction), was mit dem Erwerb neuen Wissens über eine Kultur und dem Einsatz dieses Wissens im Kommunikationsprozess verbunden ist. Dazu gehört die Fähigkeit, non-verbale Interaktionselemente zu identifizieren und diese angemessen in der Praxis anzuwenden - unter Berücksichtigung des Kommunikationskontextes. Auf der Ebene der Handlungen hat Sercu (ebd.) ebenfalls kritisches Kulturbewusstsein und politische Ausbildung (eng. critical cultural awareness/political education) angeführt. Es handelt sich nicht um die Fähigkeit, Werte, Praktiken und kulturelle Perspektiven zu identifizieren, sondern um die Fähigkeit Aspekte eigener und fremder Kultur anhand eindeutig definierter Kriterien kritisch zu beurteilen.

Das vorgestellte Modell könnte als ein Referenzmodell für die Messung interkultureller Kompetenz angenommen werden. Es sorgt nämlich eindeutig für die genauere Festlegung der Anforderungen interkultureller Kompetenz, die als Kriterien der Messung interkultureller Kompetenz sowie als Grundlage für die Auswahl entsprechender Leistungsmessungstechniken betrachtet werden können.

\section{BESONDERHEITEN DER MESSUNG INTERKULTURELLER KOMPETENZ}

Leistungsmessung, auch im Falle interkultureller Kompetenz, muss natürlich in erster Linie die traditionellen Fähigkeiten (d.h. solche, die in Schulen vermittelt werden) beschreiben. Es ist darüber hinaus aber von grundle- 
gender Bedeutung, dass die Messung auch für außerordentliche Lebensläufe, nicht konformistische Erfahrungen und Strategien, die außerhalb des normalen Qualifizierungsprozesses erworben wurden, Platz bietet (vgl. Bandura 2000). Es müssen somit auch bestimmte Merkmale der individuellen Erfahrung zum Ausdruck kommen und beschrieben werden können und zwar ordentlich und systematisch, so dass sie verglichen werden können, ohne das dies zu einer Standardisierung dieser Fähigkeiten führen würde. Diese sich aus allgemeinen Evaluationsprinzipien ergebende Schwierigkeit kommt bei der Messung interkultureller Kompetenz auch bei der Beurteilung von vier Sprachfertigkeiten deutlich zum Tragen, denn den außerschulischen/außeruniversitären Erfahrungen kommt bei der Entwicklung interkultureller Kompetenz eine nicht unerhebliche Bedeutung zu. Die Messung interkultureller Kompetenz ist nach Byram und Zarate (1997: 12) auch durch weitere Besonderheiten gekennzeichnet:

- Um das Phänomen der interkulturellen Kompetenz beschreiben und erfassen zu können, müssen Zusammenhänge zwischen der Fremdsprachendidaktik und der Soziologie/Anthropologie hergestellt werden,

- Interkulturelle Kompetenz ist somit eine interdisziplinäre Erscheinung und muss folglich auch interdisziplinär beschrieben werden. Dabei muss die Kohärenz des Begriffs gewahrt werden, der nicht einfach mit den Merkmalen einer bestimmten Disziplin gleichgesetzt werden darf,

- Jede Kompetenzmessung, die die Persönlichkeit des Lernenden oder seine psychologische Entwicklung in einem anderen als dem kognitiven Bereich widerspiegelt, wirft die Frage nach der ethischen Zulässigkeit auf, da die Messung ideologisch motiviert sein könnte.

Zum einen ergibt sich daraus, dass die interkulturelle Kompetenz nicht eindeutig quantifizierbar also nicht in exakten Zahlen zu messen ist. Doch eine Aussage über die Tendenzen ist immer möglich und berechtigt - nachstehend werden wir erwägen, mit welchem Instrumentarium bzw. mit welchen Messverfahren dies möglich ist. Zum anderen führt die Messung interkultureller Kompetenz zu einem anderen Dilemma: ist sie, zumindest im Bereich der Einstellungen, aus ethischen Gründen legitim? Auch diese Frage versuchen wir nachstehend $\mathrm{zu}$ vertiefen.

\section{MESSUNG INTERKULTURELLER KOMPETENZ - INSTRUMENTARIUM}

Mit welchen Techniken könnten die dargestellten Dimensionen der interkulturellen Kompetenz evaluativ erfasst werden? Summarische Sprachtests enthalten Aufgaben zum Vokabular, zur Grammatik, zum Hör- und Leseverständnis und zum Schreiben. Wie bereits erwähnt, kann die interkulturelle Kompetenz nicht in genauen Zahlenwerten erfasst werden, es liegt 
aber nahe, dass Tests zur Messung interkultureller Kompetenz auch Aufgaben enthalten könnten, bei denen die Testperson ihr Wissen und ihre interkulturellen Fähigkeiten unter Beweis stellen kann. Im Folgenden werden beispielhaft, ohne Anspruch auf Vollständigkeit, die in der einschlägigen Literatur und in der Fremdsprachendidaktik thematisierten Techniken der Messung einzelner Dimensionen interkultureller Kompetenz dargestellt (Byram / Zarate 1997, Bandura 2000, Facciol / Kjartansson 2003, Sercu 2005). Zur Verfügung stehen dabei grundsätzlich alle innerhalb der Fremdsprachendidaktik entwickelten Techniken. Die beim professionellen Assessment interkultureller Kompetenz verwendeten Techniken scheinen vor allem aufgrund ihrer Komplexität weniger geeignet zu sein 1 . Sie weisen zwar den Vorteil auf meist als integrative Tests konzipiert zu sein und eine holistische Beurteilung der interkulturellen Kompetenz zu ermöglichen, ihr Einsatz würde jedoch weit über die Möglichkeiten der Ausbildungsinstitutionen hinausgehen. Zunächst sollen alle Techniken tabellarisch veranschaulicht werden:

Tabelle Nr. 2: Techniken zur Messung interkultureller Kompetenz (eigene Darstellung)

\begin{tabular}{|c|c|}
\hline Ebenen der IK & Messtechniken \\
\hline Wissen & $\begin{array}{l}\text { Multiple-Choice-Aufgaben, } \\
\text { Ja/Nein-Aufgaben, } \\
\text { Essay, Quiz, } \\
\text { Selbstbewertung }\end{array}$ \\
\hline Einstellungen & $\begin{array}{l}\text { langfristiges Projekt, } \\
\text { Portfolio, } \\
\text { Fragebögen, } \\
\text { Selbstbewertung, } \\
\text { Tagebuch, } \\
\text { aufgabenorientierte Tests }\end{array}$ \\
\hline Handlungen & $\begin{array}{l}\text { langfristiges Projekt, } \\
\text { Portfolio, } \\
\text { Fragebogen } \\
\text { Selbstbewertung, } \\
\text { Tagebuch, } \\
\text { aufgabenorientierte Tests, } \\
\text { Critical-Incident-Aufgaben }\end{array}$ \\
\hline
\end{tabular}

1 Es handelt sich vor allem um die sog. interkulturellen Assessment-Center, die sowohl das punktuelle und das systemisch interkulturelle Assessment anbieten. Es werden dort im wesentlichen individuelle und soziale Teilkompetenzen überprüft, die für einen internationalen Einsatz der Führungskräfte als besonders wichtig erachtet werden. Dabei wird in der Regel auf den aus konventionellen Assessment Centern bekannten, aber stark ausgebauten Übungstypen wie Präsentationen, PostkorbAufgaben, gesteuerte/ungesteuerte Diskussionen, Erarbeiten von Problemlösungen im Team etc. aufgebaut. Assessment-Center nutzen auch ausgebaute Tests, die sie als statistisch zuverlässige Instrumente zur Messung interkultureller Sensitivität empfehlen (mehr dazu u.a. Baumer 2004: 160-208). 


\section{EVALUATION DER WISSENSEBENE INTERKULTURELLER KOMPETENZ}

Es wurde bereits mehrmals versucht, diese Ebene der interkulturellen Kompetenz (vormals: sog. Kulturwissen) durch Tests zu erfassen (u.a. Lado 1971; Valette 1986). Es eignen sich hierzu geschlossene Fragen, Lückentexte, Quiz und offene Fragen mit Vergleichsaspekten (z.B. Falk 1993: 15). Grundsätzlich erfüllen das Quiz und die anderen genannten Techniken, vorausgesetzt sie werden entsprechend angepasst, alle Anforderungen zur Ermittlung interkultureller Kompetenz auf der Wissensebene: sie können mehrperspektivisch angelegt sein, die eigene Realität mit einbeziehen etc. Es stellt sich allerdings die Frage nach dem Inhalt solcher Tests. Wenn das Testergebnis Aufschluss darüber geben soll, ob eine Person fähig ist, mit Personen aus einem anderen Kulturraum auf eine interkulturell kompetente Art und Weise zu interagieren, liegt die Schwierigkeit der Bewertung dieser Ebene der interkulturellen Kompetenz in der richtigen Auswahl relevanter Wissenselemente. Das traditionelle landeskundliche Curriculum beinhaltet einen Wissensumfang, der viele, jedoch bei weitem nicht alle, kulturelle Bereiche abdeckt. Diese Bereiche sind einerseits thematisch geordnet, andererseits umfassen sie unterschiedliche Lebensebenen (Institutionen, Alltag etc.).Vor diesem Hintergrund muss eindeutig festgestellt werden, dass im Rahmen der Messung interkultureller Kompetenz unmöglich alle Bereiche berücksichtigt werden können. Mit anderen Worten: die Fremdkultur/Eigenkultur kann in ihrer Gesamtheit bei der Testkonstruktion kaum den Bezugspunkt bilden. Es kommt aber ein weiterer Aspekt hinzu, der in Betracht gezogen werden muss: Während die Sprachkompetenz bestimmte Vorkenntnisse voraussetzt, die bei ihrer Beurteilung auch überprüft werden können, wird man mit der Vorgehensweise der Messung des Kulturwissens nicht zum gewünschten Ergebnis kommen (so ist es z.B. schwer festzustellen, welche Vorkenntnisse das Wissen über politische Aspekte einzelner Bundesländer voraussetzt). Dies ist nicht zuletzt auf das moderne, offene Kulturverständnis zurückzuführen². Es wäre somit wenig sinnvoll einzelne, miteinander nicht verbundene Tatsachen abzufragen.

Seit den 70er Jahren hat sich in der Fremdsprachendidaktik die Idee der Lernerorientierung (Lernerzentriertheit) durchgesetzt ${ }^{3}$. Gemäß diesem Grundsatz können die kulturellen Informationen als relevant gelten, die der Lernende für das Agieren in seinem Interessenbereich benötigt. Mit anderen Worten muss der Wissensumfang für eine bestimmte Lernergruppe von Nutzen und von Interesse d.h. auf diese zugeschnitten sein. Diese These soll

${ }^{2}$ Hierzu wird auf die Netzwerkmodelle der ersten und der zweiten Moderne von Bolten (2004: $50 \mathrm{f}$ verwiesen, die diese Denkweise widerspiegeln.

${ }^{3}$ Z.B. Bausch et al. (Hrsg.) 1982. 
den Ansatzpunkt für die Auswahl der zur Entwicklung und anschließenden Evaluation interkultureller Kompetenz auf der Wissensebene relevanten Inhalte bilden (z.B. bei Fremdsprachelehrern in Polen wären es die Wissensbereiche, die ein Lehrer beherrschen sollte, um in den drei grundlegenden Schultypen in Polen interkulturelle Kompetenz im Bereich Deutsch als Fremdsprache vermitteln zu können).

Zusammenfassend ist festzuhalten, dass sich die Messung interkultureller Kompetenz auf der Wissensebene nicht auf konkrete Tatsachen stützt, sondern auf die Einschätzung bestimmter Wissensbereiche, die eine konkrete Person in Bezug auf ihre Bedürfnisse beherrschen sollte.

\section{MESSTECHNIKEN AUF HANDLUNGSEBENE INTERKULTURELLER KOMPETENZ}

Zur Messung interkultureller Kompetenz auf der Handlungsebene werden sowohl traditionelle Testformen der punktuellen Leistungsmessung als auch Testarten, die einen formativen Charakter haben eingesetzt (vgl. Kramsch 1993: 265f., Anlage II; Little/Simpson 2003: 19 nach Sercu 2005: 13f.). In dem ersten Fall handelt es sich um textorientierte Aufgaben, bei denen die Testteilnehmer zeigen, dass sie fähig sind, die eigene/fremde Kultur zu erforschen, Informationen über eigen-/fremdkulturelle Merkmale zu sammeln und diese Informationen kohärent in der fremden Sprache zu präsentieren. Im zweiten Fall wird ein Portfolio als Messwerkzeug betrachtet (hierzu: Sercu 2005: 13f.) $)^{4}$

Der Vorschlag der aufgabenorientierten Leistungsmessung von Kramsch setzt eine Bearbeitung von "kritischen“ Situationen mit dem Ziel voraus, praktische Probleme zu lösen. Der Testteilnehmer muss dabei auf seine Kenntnisse zurückgreifen und diese praktisch einsetzen. Die vom Testteilnehmer unternommene genaue Analyse einer kritischen Situation gibt Einsicht in seine Bewältigungs- und Verarbeitungsstrategien. Das angeführte Beispiel baut auf der Theorie der critical incidents (Göbel 2001) auf. Die Critical-Incident-Methode könnte aus den oben angeführten Gründen auch bei der Messung interkultureller Kompetenz in der Fremdsprachendidaktik angewandt werden. Für die Erstellung der Testaufgaben würde dabei auf ein kritisches Ereignis (schwierige Situation, critical incident) zurückgegriffen werden, dessen Analyse die Antworten der Testteilnehmer in die Entwicklungsstufen interkultureller Kompetenz klassifizieren ließe. Da aber keine umfangreiche Sammlung von kritischen Situationen vorhanden ist, bedarf

${ }^{4}$ Sercu führt auch mehrere Kriterien auf, die ein als Messwerkzeug für die interkulturelle Kompetenz gedachtes Portfolio zu erfüllen hat. 
die Nutzung von Critical-Incident-Aufgaben zunächst einer systematischen Erhebung von für ein Länderpaar typischen kritischen Situationen. Diese Erhebung könnte in Form von Interviews mit Personen durchgeführt werden, die solche Erfahrung gemacht haben. Dabei müssen diese Situationen immer den spezifischen Anforderungen entsprechen, nach einheitlichen Kriterien erhoben werden und Lösungsmöglichkeiten zulassen, die die Kategorisierung der Antworten ermöglichen.

\section{ERFASSUNG DER EINSTELLUNGEN - EIN DING DER UNMÖGLICHKEIT?}

$\mathrm{Zu}$ den Besonderheiten der Messung interkultureller Kompetenz gehört sicherlich die Erfassung der Einstellungen. Diese wirft die wohl umstrittenste Frage im Zusammenhang mit der interkulturellen Kompetenz auf: Sind die Einstellungen überhaupt evaluativ erfassbar und ist ihre Evaluation legitim.

Bei der Beantwortung der Frage zur Zulässigkeit der Evaluation von Einstellungen kommt folgender Gedankengang voll zum Tragen: Leistungsmessung muss nicht immer ausschließlich der Beurteilung dienen, sondern kann auch zur Feststellung eines bestimmten Sachverhalts zum Einsatz kommen. Am besten drückt es Byram $(1997,2000)$ aus, der die Ansicht vertritt, dass die Zulässigkeit einer Evaluation strittig ist, wenn sie auf die Beurteilung einer bestimmten Person abzielt. Wird die Evaluation jedoch "nur" vorgenommen, um eine Momentaufnahme der Kompetenzen zu machen, hat sie nichts Strittiges an sich (Byram 2000: 9). In diesem Sinne ist auch die Messung der Einstellungen (wie bei der interkulturellen Kompetenz der Fall) legitim.

Dass die Evaluation von Einstellungen auch möglich ist, bezeugen viele fundierte Untersuchungen z.B. zu Stereotypen. Über diese Art der Messung berichten auch Böhner und Wänke (2004) ausführlich, die explizit von der Änderung der Einstellungen sprechen. Byram und Zarate (1994: 138) stellen sich angesichts der Schwierigkeiten der Messung von Einstellungen folgende Frage:

Can we simply ignore the assessment of attitudes and the educational purpose associated with them? To do so and to remain consistent, we would have to abandon statements which suggest that language and culture teaching is intended to stimulate positive attitudes.

Byram verfolgt somit auf dieser Ebene den Grundsatz: what is not assessed is not taught, allerdings schlägt er vor, den Zeitpunkt der Messung von Einstellungen zu verschieben (Byram/Zarate 1994: 138): „(...) we can delay assessment until we can reasonably suppose that learners have reached an appropriate stage of psychological development ${ }^{\prime \prime}$.

Eindeutig wird hier auf den Zeitpunkt verwiesen, zu dem die Fähigkeit eines Menschen zur Selbstevaluation entwickelt ist. An dieser Stelle er- 
scheint es sinnvoll, in einem Exkurs in die Entwicklungspsychologie zu ermitteln, wann "vernünftig angenommen" werden kann, dass eine Person eine zur Selbsteinsicht und Selbstevaluation befähigende Stufe der kognitiven Entwicklung erreicht hat.

In der psychologischen Fachliteratur wird oft betont, dass die Grenzen zwischen einzelnen Entwicklungsphasen eines Individuums schwer festzulegen sind und die Altersangaben in diesem Zusammenhang nur einen approximativen Charakter haben. Sęk/Sommerfeld (1990) stellen einen Kata$\log$ von Eigenschaften, die zur Selbstreflexion und zur Selbsteinschätzung eigener Einstellungen befähigen, erst für Menschen auf, die in den unterschiedlichen Typologien der menschlichen Entwicklung als junge Erwachsene bezeichnet werden (z.B. Obuchowska 2000: 166). Zu diesen Eigenschaften gehören nach Sęk/Sommerfeld (1990) ein ausgeprägtes Identitätsgefühl, Autonomiebedarf, die Bereitschaft, für sich selbst und andere Verantwortung zu übernehmen, Entwicklung einer konkreten Vorstellung vom Sinn des Lebens, die Fähigkeit andere Menschen zu verstehen, die Fähigkeit sich emotional an einen anderen Menschen zu binden, die Fähigkeit, die Aufgaben des Lebens zu meistern und Ambiguitäten zu bewältigen (Übers. A.B.). Es kann somit angenommen werden, dass diese Entwicklungsphase erst bei Studierenden im akademischen Sprachunterricht vorausgesetzt werden kann. Bei diesen Fremdsprachenlernenden wäre also der Self-AssessmentFragebogen als ein angebrachtes Werkzeug der Leistungsbewertung vorzuschlagen. Dabei kann dieses Instrument sowohl punktuell als auch über einen längeren Zeitraum eingesetzt werden. Sercu (2005: 14) vertritt die Meinung, dass solche Fragebögen ein umfassendes Instrument darstellen und sich auch zur Messung der übrigen Ebenen der interkulturellen Kompetenz eignen. Ein Beispiel für eine mit Hilfe dieses Instrumentes erfolgte Messung interkultureller Kompetenz ist im Rahmen des Bergen Cando Projects ${ }^{5}$ vom European Centre for Modern Languages in Graz entwickelt worden.

\section{ABSCHLUSS}

Die Vorgehensweise bei der Messung interkultureller Kompetenz bewegt sich, wie gezeigt, im Erwartungsfeld zeitgenössischer Evaluationsverfahren zwischen zwei funktionalen Polen: der punktuellen Leistungsmessung (summative Funktion) und den Verfahren, durch die UrsacheWirkung-Zusammenhänge auch im Bereich interkultureller Kompetenz laufend erschlossen werden können (formative Funktion). Somit steht der Evaluation interkultureller Kompetenz eine bereite Palette an Möglichkeiten

${ }^{5}$ http://www.ecml.at/cando/files/start.htm (Stand: 27. Mai 2007) 
zur Verfügung, die jedoch auf bestimmte Grenzen stoßen, welche nachstehend zusammengefasst werden sollen:

- Die formativen Evaluationsformen, die weniger formalisiert als traditionelle Tests sind, sich aber unter Berücksichtigung der Besonderheiten interkultureller Kompetenz zu derer Messung mehr eignen, nehmen mehr Zeit in Anspruch und können kaum einmalig angewandt werden. Sie sind also insgesamt weniger praktisch, was in der institutionalisierten Lehre ein großer Nachteil ist. Manche formative Testformen - wie Selbstevaluation - bedürfen darüber hinaus der Fähigkeiten, über die ein Individuum erst auf einer bestimmten Entwicklungsstufe verfügt.

- Wichtig ist, dass die Begrenztheit der Aussagekraft einzelner Test/Messtechniken im Hinblick auf die Besonderheiten interkultureller Kompetenz eingestanden wird und als Ziel der Leistungsmessung ein möglichst konsistentes Gesamtbild aus verschiedenen Testverfahren angestrebt wird. Bei der Messung interkultureller Kompetenz ist die Triangulation von verschiedenen Leistungsmessungstechniken erforderlich.

- Als Ergebnis der Messung interkultureller Kompetenz sind kaum exakte Zahlen zu erwarten, viel mehr soll anhand der angewandten Techniken der Leistungsmessung eine Tendenzbeurteilung abgegeben werden.

Abschließend sei noch einmal betont, dass das Reflektieren über die Grenzen der Messung interkultureller Kompetenz der Vornahme einer solchen Bewertung ihre Legitimität keinesfalls abspricht. Viel mehr soll die Messung interkultureller Kompetenz in deren Möglichkeiten und Grenzen zum besseren Verständnis der Evaluation betragen, die nicht der Beurteilung einer bestimmten Person dienen, sondern uns - anhand der Analyse der Leistungsmessungsergebnisse - vermehrtes Wissen über interkulturelle Kompetenz selbst, sowie die Effektivität der Methoden ihrer Vermittlung bieten soll, denn erst eine auf diese Weise aufgefasste Evaluation mündet in verfeinerter Sensibilität im Umgang mit den Mitmenschen.

\section{LITERATURVERZEICHNIS}

Bandura, E., 2000, Ewaluacja kompetencji interkulturowej. In: H. Komorowska, Ewaluacja w nauce języka obcego. Bialystok: Wydawnictwo Uniwersytetu w Białymstoku: 11-23.

Baumer, T., 2004, Handbuch interkulturelle Kompetenz. Bd. 2. orell flüssli Zürich: Verlag AG.

Bausch, K.-R./Christ, H./Hüllen, W./Krumm, H.-J., (Hrsg.), 1982, Das Postulat der Lerrnerzentriertheit: Rückwirkungen auf die Theorie des Fremdsprachenunterrichts. Heidelberg Julius Groos.

Boeckmann, K.-B., 2006, Dimensionen von Interkulturalität im Kontext des Fremd- und Zweitsprachenunterrichts, Zeitschrift für Interkulturellen Fremdsprachenunterricht 11, available: http://www.spz.tu-darmstadt.de/projekt_ejournal/jg-11-3/beitrag/Boeckmann1.htm 
Bolten, J., 2001, Interkultureller Assessment-Center. In: W. Sarges (Hrsg.), Weiterentwicklungen der Assessment Center-Methode. Göttingen u.a.: 213-218.

Bolten, J., 2004, Interkulturelle Personalentwicklung im Zeichen der Globalisierung: „Paradigmenwandel" oder "Paradigmenkorrektur"? In: J. Bolten (Hrsg.), Interkulturelles Handeln in der Wirtschaft. Verlag Wissenschaft \& Praxis, Sternenfels: 40-62.

Böhner, G./Wänke, M., 2004, Postawy i zmiana postaw. Gdańsk: Gdańskie Wydawnictwo Psychologiczne.

Byram, M., 1997, Teaching and Assessing Intercultural Communicative Competence, Clevendon: Multilingual Matters.

Byram, M., 2000, Assessing Intercultural Competence in Language Teaching. In: Sprogforum, Nr. 18, Vol. 6: 8-13.

Byram, M./Zarate, G., 1997, Definitions, objectives and assessment of sociocultural competence. In: M. Byram/G. Zarate/G. Neuner, Sociocultural competence in language learning and teaching. Strasbourg Council of Europe Publishing: S. 9-46.

Falk, R., 1993, Spotlight on the USA. Oxford/New York: Oxford University Press.

Facciol, R./Kjartansson, R., 2003, Testing times: testing intercultural communicative competence. In: I. Lázár, Incorporating intercultural communicative competence in language teacher education. Strasbourg: Council of Europe Publishing: 73-106.

Göbel, K., 2001, Die Bedeutung der Analyse interkultureller Konfliktlösestrategien für die interkulturelle Erziehung in der Schule: Forschungsergebnisse einer Akkulturationsstudie in Chile. In: U. Wagner (Hrsg.), Interkulturalität im Arbeitsfeld Schule. Opladen, Leske + Budrich: 161-177.

Lado, R. (1971): Testen im Sprachunterricht. Handbuch für die Erstellung und den Gebrauch von Leistungstests im Fremdsprachenunterricht. Aus dem Amerikanischen von R. Freudenstein. München. (Original 1961).

Müller, B.-D., 1994, Fremdsprachenunterricht als Ausgangspunkt für interkulturelles Lernen. In: K.-R. Bausch/H. Christ/H.-J. Krumm (Hrsg.), Interkulturelles Lernen im Fremdsprachenunterricht. Tübingen: 155-164.

Obuchowska, I., 2000, Adolescencja. In: B. Harwas-Napierała, J. Trempała, Psychologia rozwoju człowieka, t. 2, Warszawa: Wydawnictwo Naukowe PWN: 163-199.

Sercu, L., 2004, Assessing intercultural competence: a framework for systematic test development in foreign language educatin and beyond. In: Intercultural Education, Bd. 15, Nr. 1: 73-89.

Sercu, L., 2005, Testing intercultural competence in a foreign language. Current approaches and future challenges. In: Belgian Journal of English Language and Literatures (BELL), N. S. 3: 149-169.

Sęk, H./Sommerfeld, A., 1990, Być dorostym! Legnica: Centrum Doskonalenia Nauczycieli.

Strewe, B., 2005, Bewertung und Benotung interkultureller Kompetenzen in OnlineStudiengängen zur Interkulturellen Kommunikation. Bewertung der interkulturellen Kompetenz von Studierenden - ein Dilemma?. In: Interculture-Online 11/2005. Available: http://www.interculture-online.info/index.php?bereich=articles

Thomas, A., 2003, Interkulturelle Kompetenz - Grundlagen, Probleme und Konzepte. In: Erwägen, Wissen, Ethik (EWE), 14, H. 1, S. 137-150.

Valette, R.M., 1986, The culture test. In: M.J. Baldees, Culture Bound. Bridging the cultural gap in language teaching. Cambridge: Cambridge University Press: 179-197.

Vollmer, H., 2003, Leistungsmessung, Lernerfolgskontrolle und Selbstkontrolle. In: K.-R. Bausch /H. Christ/H.-J. Krumm, Handbuch Fremdsprachenunterricht. Tübingen/Basel, 171-175. 